\title{
Cesare Segre - Philologie italienne et critique génétique
}

Entretien avec Maria Teresa Giaveri (en collaboration avec Erica Durante)

\section{Cesare Segre et Maria Teresa Giaveri}

\section{OpenEdition}

Journals

Édition électronique

URL : https://journals.openedition.org/genesis/100

DOI : 10.4000/genesis. 100

ISSN : 2268-1590

Éditeur :

Presses universitaires de Paris Sorbonne (PUPS), Société internationale de génétique artistique littéraire et scientifique (SIGALES)

\section{Édition imprimée}

Date de publication : 20 juin 2010

Pagination : 25-27

ISBN : 978-2-84050-697-3

ISSN : $1167-5101$

\section{Référence électronique}

Cesare Segre et Maria Teresa Giaveri, « Cesare Segre - Philologie italienne et critique génétique », Genesis [En ligne], 30 | 2010, mis en ligne le 17 mai 2013, consulté le 30 mars 2023. URL : http:// journals.openedition.org/genesis/100; DOI : https://doi.org/10.4000/genesis.100 


\title{
Philologie italienne et critique génétique Entretien avec Maria Teresa Giaveri (en collaboration avec Erica Durante)
}

\author{
Cesare Segre
}

Pour avoir une idée de la situation actuelle des études de genèse en Italie, nous avons interrogé l' un des plus éminents représentants actuels de la philologie italienne, Cesare Segre, professeur émérite de l'université de Pavie, fondateur et président du Centro di Ricerca dei Testi e Tradizioni testuali (Pavie). Cesare Segre a renouvelé la théorie ecdotique par ses études et par ses éditions de la Chanson de Roland, du Bestiaire d'Amours et de l'Orlando furioso.

Maria Teresa Giaveri - La critique génétique française, née au sein du Centre d'Analyse des Manuscrits modernes (CAM) s'est rapidement développée dans le cadre de l'Institut des Textes et Manuscrits modernes (ITEM), qui est ainsi devenu l' une des unités de recherche les plus dynamiques du CNRS. En Italie, malgré la longue tradition d'analyse des manuscrits et les différentes initiatives spécialisées, on n'a pourtant vu naître aucun organisme similaire. Est-ce un pur hasard ou, plutôt, une occasion qui permet de réfléchir sur la différence des structures de recherche dans chacun des deux pays?

Cesare Segre - En Italie, le Centre national des Recherches (CNR) a mis au concours très peu de postes de chercheurs ; je ne suis d'ailleurs au courant que de postes en psycholinguistique. Ce manque de recrutements, qui n'est pas sans rapport avec l'absence, en Italie, d'un institut tel que l'ITEM, explique que, dans la péninsule, les études génétiques ne soient pas organisées et structurées autour d'un centre institutionnel. En effet, les recherches de type génétique sont menées dans différentes structures et différentes régions d'Italie; elles n'ont jamais connu de véritable questionnement sur la « critique génétique », et sont toujours issues de la nécessité de travailler sur des auteurs précis. Cela veut dire que, si un chercheur souhaite travailler par exemple sur Dino Campana, dont sont conservés les brouillons, il mènera une étude ou une édition génétique sur l'œuvre de cet auteur. En d'autres termes, en Italie, personne ne se déclare spécialiste de génétique des textes littéraires ; certains chercheurs peuvent se dire spécialistes de tel ou tel écrivain, voire être auteurs d'une étude (ou d'une édition) génétique sur l'œuvre de tel ou tel écrivain. En règle générale, on part plutôt de certains résultats obtenus, pour mettre en place, en fonction des besoins, les moyens qui permettent de mener à bien un programme de recherche. Aussi, bien qu'il existe un nombre important de spécialistes des manuscrits littéraires, on peut affirmer que, dans l'ensemble, la génétique n'a finalement qu'une assez faible visibilité.

M. T. G. - En septembre 1990, nous avons organisé à Gargnano, aux bords du lac de Garde, une rencontre entre généticiens de l'ITEM et spécialistes italiens de la "critique des variantes ${ }^{1}$ ». À l'époque, nous ne nous connaissions pas bien les uns les autres, mais au cours de ce colloque, nous avons vu apparaître un certain nombre d' analogies évidentes mais aussi des différences de parcours et de traditions entre Italiens et Français. À cette occasion, vous aviez d'ailleurs vous-même symboliquement accueilli les participants dans la salle d' anatomie de l' université de Pavie, où s' est tenue la séance d' ouverture du colloque. Depuis, d'autres rencontres ont fait suite à cette première, par exemple celle qui s'est déroulée à Paris en mars 1994, au Centre culturel italien, à l'occasion de la présentation des actes de ce même colloque ${ }^{2}$. Ces rencontres ont marqué une pénétration progressive en

1. Pour les actes du colloque, voir le volume I sentieri della creazione : tracce, traiettorie, modelli. Les Sentiers de la Création : traces, trajectoires, modèles, dir. Maria Teresa Giaveri et Almuth Grésillon, Reggio Emilia, Diabasis, 1994. [N.d.T., Erica Durante]

2. Voir le texte de la conférence de Cesare Segre : «Critique des variantes et critique génétique », Genesis, n 7, p. 29-46. [N.d.T.] 
Italie du lexique introduit par l'ITEM (notamment la diffusion de termes comme "avant-texte », "génétique », etc.). Comment retraceriez-vous ces vingt années d'échange entre nos deux traditions européennes? Quelles sont vos impressions par rapport aux recherches italiennes et françaises, à la fois communes et différentes, telles qu' elles ont été menées au long de ces vingt ans?

C. S. - Ma réponse s'inscrit dans le sillage du panorama que j'ai dressé en répondant à la première question. Au cours des dernières années ont été menés en Italie de nombreux travaux qui témoignent d'une expérience plus fine et plus consciente des études génétiques, mais qui n'envisagent pas pour autant une coordination ni un aboutissement théorique. Seule exception, rappelons-le, le chapitre digne de louanges d'Alfredo Stussi, intitulé «Filologia d'autore », paru dans son manuel de philologie italienne (Introduzione agli studi di filologia italiana, Bologne, Il Mulino, 1994). Notons par ailleurs la persistance d'une terminologie «nationale » (critica delle varianti, filologia d'autore), et la méfiance, y compris théorique, vis-à-vis de la notion de « genèse », qui, me semble-t-il, est en réalité peu heureuse, bien que ce soit maintenant le terme consacré. D'un point de vue théorique, on a l'impression que le fait de se limiter à l'étude des autographes et des brouillons revient à négliger les variantes rédactionnelles dans les éditions imprimées et les corrections éditoriales dont l'étude relève de la filologia dei testi a stampa (« philologie de l'imprimé »). En règle générale, je dirais que la philologie romane, bien vivante chez nous, et presque inexistante en France, se situe au cœur de ce travail, et cela dans l'indifférence des autres disciplines, notamment littéraires. Tout cela implique, de manière naturelle, que la critique génétique vient s'insérer dans un cadre très vaste, à la fois en termes de résultats obtenus par la philologie et d'histoire de la discipline.

M. T. G. - On peut avancer que la critique génétique s'est développée en France en opposition à la tradition philologique, alors que, en Italie, elle est née, pour ainsi dire, d'une côte de la philologie. S'agit-il de deux disciplines différentes ou bien d' une seule et unique discipline avec des bifurcations possibles? Quelle est votre opinion à ce sujet?
C.S. - Ce que vous affirmez dans votre question est tout à fait exact, et, en tant que philologue, je ne peux qu'approuver cette orientation, d'ailleurs inévitable dans le contexte culturel italien. La critique génétique ne peut que se servir de l'expérience séculaire des philologues; de son côté, la philologie ne peut pas faire la sourde oreille à la très riche problématique découverte et approfondie par la critique génétique.

M. T. G. - Je voudrais enfin vous demander de présenter aux lecteurs de Genesis le Centro di Ricerca dei Testi e Tradizioni testuali que vous avez vous-même fondé à l'université de Pavie et dont vous êtes le président.

C. S. - Les philologues, tout comme les généticiens, font référence à deux concepts fondateurs, celui de texte et celui de variante. Or, si le texte est un concept si ample qu'il requiert des précisions et des distinctions plus fines, la notion de variante est consubstantielle à chacune de nos expériences. La variante, nous le savons, peut être attribuée à l'auteur du texte ou à l'élaboration anonyme de la tradition. Le philologue doit (et en cela consiste sa première tâche) distinguer les variantes d'auteur de celles de la tradition, mais il doit aussi savoir que la tradition ellemême transmet parfois des variantes d'auteur. Ces deux types de variante peuvent également être présents à l'intérieur d'un même texte, et il faut immédiatement les différencier (je pense au cas des Satires de l'Arioste, transmises par un codex idiographe, c'est-à-dire transcrit par un copiste sous le contrôle de l'auteur). Dans un cas pareil, je me demande si l'on peut distinguer de manière nette le travail du philologue de celui du généticien; il me semble que non. Et, que dire des variantes d'auteur transmises par les textes latins, à travers des transcriptions qui ne sont pas de l'auteur ? C'est dans cette perspective que se situe la réflexion du Centre que je dirige à Pavie et qui est ouvert aux recherches les plus diverses sur les textes, mais aussi aux différentes philologies, allant de la philologie classique à la philologie appliquée, en passant par la linguistique (n'oublions pas la Textlinguistik), et portant une attention particulière à la critique des variantes d'auteur et à la génétique. Afin de donner une idée de la portée de nos projets, je citerai la première initiative du Centre, à savoir une Concordance diachronique du Roland furieux, qui 
compare, vers par vers, les corrections introduites par l'Arioste dans ses trois éditions successives $(1516,1521$ et 1532), pour un total de 38736 vers. À court terme, j'envisage également de promouvoir un «Observatoire de la critique génétique » en Italie, qui vient d'être créé afin de recenser et collecter toutes les contributions, articles et éditions s'inscrivant dans le domaine de la critique génétique. Cela facilitera l'échange et la collaboration (déjà existants) avec l'ITEM. Cependant, la réussite de cet «Observatoire » dépendra entièrement de l'engagement, du dynamisme et de la volonté des chercheurs qui participent à nos projets. 